\title{
The Case Against Delusional Parasitosis
}

\author{
Omar M. Amin \\ Parasitology Center Inc. (PCI), 11445 E. Via Linda \# 2-419, Scottsdale 85259, Arizona
}

Received: August 29, 2014 / Accepted: September 30, 2014 / Published: September 30, 2014.

\begin{abstract}
The body of knowledge on delusional parasitosis incriminates "delusional patients" as having imaginary external and/or internal symptoms that feel like parasite movements, dismisses them as psychiatric cases, and confuses cause with effect. The author is critical of these arguments and believes that the symptoms of crawling and pin-pricking are genuine; just misinterpreted as parasite movements. Based on the author's research on over 1000 "delusional" patients since 1996 at the Parasitology Center, Inc. (PCI), the author has established that the symptoms of crawling and pin-pricking are caused by toxicity from exposure to incompatible dental materials, implants, environmental toxins among others that alter the propagation of normal nerve impulses. The author has also observed that external parasites/organisms including springtails (Collembola), other arthropods, bacteria, and fungi represent only opportunistic infections of skin sites compromised by the toxicity disorder involving the elimination of toxins through the skin. The author has described a new pathological disorder, Neuro-cutaneous Syndrome (NCS) primarily associated with dental toxicity to which "delusional parasitosis" and Morgellons disease cases could be assigned. The author has developed a protocol for the resolution of the symptoms of NCS cases. When followed to the letter, all symptoms of NCS, conventionally called delusional parasitosis, are invariably and irreversibly resolved; each individual's unique circumstances taken into account. The author is also presenting a detailed case history of an NCS patient that has recovered from her experiential personal perspective.
\end{abstract}

Key words: Neuro-cutaneous Syndrome (NCS), Morgellons, dental toxins, delusional parasitosis, case history.

\section{Introduction}

There is ample literature describing the reported symptoms of delusional parasitosis cases and characterizing patients as psychiatric cases [1-7]. The two most recent articles are those of Shelomi [8], an entomologist who also reported on a case of photo manipulation of presumed springtail (Collembola: Arthropoda) infections, and Lyons [9], a veterinarian who presented 3 case histories.

Judging by the author's experience with over 1000 "delusionary" patients that the author has seen at the author's Parasitology Center, Inc. (PCI) in Arizona, however, the above reported "delusionary" cases appear to be cases of Neuro-cutaneous Syndrome (NCS). NCS is a toxicity disorder related to Morgellons disease, caused mostly by intimate exposure to incompatible chemicals, especially dental

Corresponding author: Omar M. Amin, Ph.D., professor, research fields: parasitology, infectious diseases. E-mail: omaramin@aol.com. materials [10-21]. Other toxic exposures are occasionally involved. Proponents of the delusional parasitosis convention do not appear to have stopped to ask the following question: what if the main symptoms of crawling and pin-pricking declared by "delusional" patients are real; just misinterpreted as parasite movement. The author has asked that question, and during 15 years of research, the author has come up with an alternative explanation that works.

The author's rebuttal to the delusional parasitosis literature referenced above, especially that of Shelomi [8] is in 4 sections. (1) Do springtails actually infest people? (2) What is delusional parasitosis really? (3) A case history debunking the delusional pseudo-diagnosis. (4) Additional notes about toxic dental materials.

\section{Materials and Methods}

Patients were interviewed and clinical and dental history were screened. Oral cavity, scalp, and skin 
were examined for signs of compromised tissues and abnormal appearances. The interview focused on any sources of toxic exposures in a timeline to establish possible associations with clinical symptoms. Patients fill out a lengthy questionnaire addressing neurological, cutaneous, organ system, oral, allergy, and general issues as well as previous dental and related clinical history and recreational drug history. Used dental materials are checked against the OSHA MSDS forms for possible pathological relationships. If the patient is diagnosed with Neurocutaneous Syndrome (NCS) or Morgellons, he/she is given a protocol involving running a dental bio-compatibility test contracted with a Colorado laboratory, dental rehabilitation and detoxification using homeopathic remedies. The biocompatibility test will determine the reactivity of the patient to known dental materials and suggest replacement chemicals to which the patient has no reactivity.

\subsection{Consent}

Written informed consent was obtained from the patient for publication of this case report and accompanying images. A copy of the written consent is available for review by the Editor-in-Chief of this journal.

\section{Results and Discussion}

\subsection{Do Springtails Actually Infect People?}

The author knows that springtails do "infect" humans. At PCI, the author collected springtails from a few patients since 1995. The author personally recovered a number of springtails from scalp lesions of $\mathrm{JH}$, a tall healthy, well-nourished middle aged white American female from Arizona in December, 1995 [22]. See pictures of 1 of JH's scalp lesions (Fig. 4) and 1 of a springtail from the same patient (Fig. 5) [22]. In another case, the author collected at least 2 springtails from a scalp lesion of $\mathrm{TH}$, a 44 years old female from Washington who was diagnosed with NCS at PCI on June 29, 2005. A bacterial culture from her chest was tested positive for Staphylococcus aureus; an opportunistic infection. Other reports of springtails collected from people, some with dermatitis, include those of Hunter et al. [23] and Scott et al. [24].

\subsection{Other Skin Infestations}

The presence of arthropods on humans, especially in dermatitis cases, is not limited to springtails. Amin [25] recovered a tick nymph, small ant, an oribatid mite, and various insect parts from facial mucoid sores of a middle aged NCS case; a female who lived in an old rural home in the woods of Oklahoma.

\subsection{Bacterial and Fungal Infections}

The author's bacterial culture swab tests from open sores of NCS cases usually show infections with Staphylococcus aureus (S. coagulase positive), S. epidermidis ( $S$. coagulase negative), or $S$. haemolyticus, as well as with metabolic waste, mycelia or sporangia of fungi such as Madurella sp., among others. The latter material from Madurella sp. appears to be related to the often reported black specks and fibers $[10,22]$. The healing of these lesions was observed to be proportional to the exit of the remaining fibers from lesions [25].

\subsection{Opportunistic Infections}

It is important to view the finding of springtails, other arthropods, bacteria, and fungi from mucoid sores/lesions of "delusional" dermatitis patients in the proper context as only opportunistic infections. None is believed to cause delusionary parasitosis, NCS, or Morgellons disease. In reality, it is the opposite. Opportunistic infections are actually attracted to skin sites of elimination of dental and other toxins. Lesions can be microscopic but still emit enough odors to attract opportunistic skin infections. Opportunistic organisms appear to feed and seek moisture, and were occasionally observed to aggregate in large numbers in those sites $[10,15,22,25$, unpublished data]. 

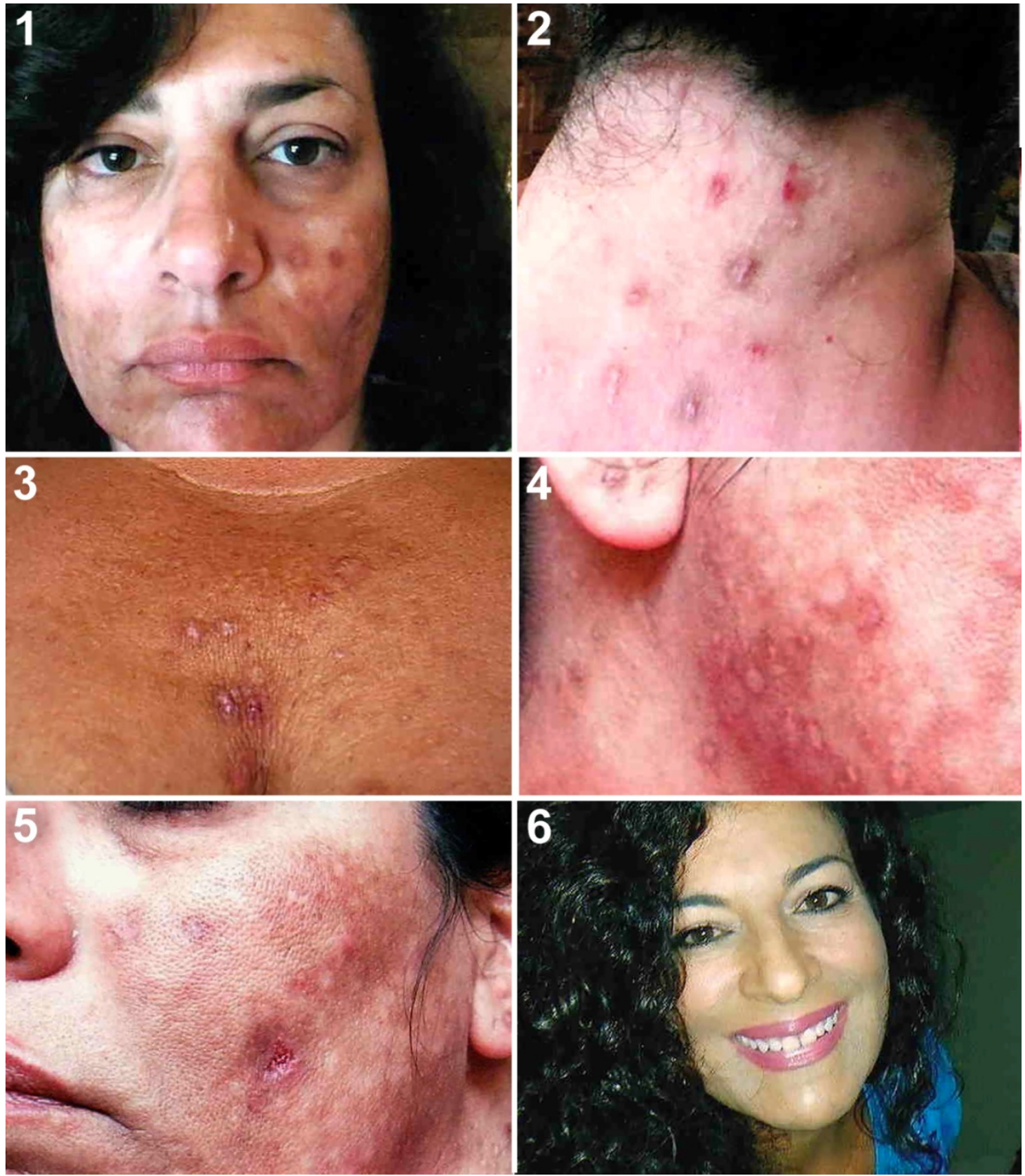

Fig. 1-6 GC during the height of symptoms in 2010 showing neck and facial lesions in various sites (Fig. 1-5) and after recovery in 2013 (Fig. 6).

\subsection{What is Delusional Parasitosis Really?}

The terms Morgellons, and Neuro-cutaneous Syndrome (NCS) as characterized by Amin [10-19, 22] are used interchangeably, yet cautiously, as their symptoms are similar to each other and to those reported in delusionary parasitosis cases. NCS was described from many patients showing dermatological and neurological disorders [10, 22]. Neurological disorders manifest as crawling and pin-pricking sensations that the author attribute to toxic corruption of normal propagation of nerve impulses but interpreted by patients as the movement of parasites in their bodies and hence the delusionary parasitosis diagnosis. The passage of toxic chemicals through the skin for elimination will cause the skin to break out 
and the resulting sores/lesions will attract opportunistic infections [10]. Of the many different possible exposures, the most common are dental materials, especially sealants, liners, bases, cements, adhesives, bonding agents, metals, crowns, and root canal materials $[10,14,20]$. The role of these dental materials as the causative agents of NCS was verified by the application of the concept of cause-effect relationship.

\subsection{Other Toxins}

The present report does not address non-dental exposures associated with NCS such as chemical fumes, cleaning agents, recreational drugs, implants, insecticides, among others.

\subsection{Cases}

The author has seen over 1000 patients to date and have developed a treatment protocol which includes detoxification and dental rehabilitation, among other measures. Patients diagnosed with NCS at the author's facility (PCI) that have used the author's protocol have invariably and irreversibly recovered; each individual's unique circumstances taken into account. See especially Amin [18] for recovered patients' accounts and the author's web site www.parasitetesting.com which includes testimonials by many other patients, a few videos, images of cases, and a complete list of the author's accessible publications on the subject, among other related topics.

\subsection{Objectives of the Research}

Because "delusional" patients were convinced that their crawling, movement and pricking sensations were caused by parasites, and because the author's thorough examinations have shown that they had no parasites, The author was determined to find out the real causes of these symptoms. A common denominator shared by practically all dental patients was incompatible dental materials that were demonstrated to have some toxic effects as per the OSHA required MSDS (material safety data sheets) forms. Establishing a cause and effect relationship was of primary importance in the design of the author's research. The various stages of this research that ultimately lead to this conclusion are documented below.

\subsection{Documentation}

At PCI, the author has been researching NCS since 1996. Because the author's research was published in holistic medical and dental journals not usually read by parasitology journals readership and because of the newness of the author's contributions to said readership, the author is providing a brief chronological listing of research done at the author's facility to resolve the delusionary parasitosis issue characterized by us as NCS. The author's early reports on this syndrome included the description of a case with many facial opportunistic infections from Oklahoma [25]. Amin [22] first named and diagnosed NCS from 3 more cases, with a special reference to fibers and springtails (Collembola). By 2003, the author was able to provide a comprehensive diagnosis of NCS and established the link with dental toxins as the primary causative agents. Amin [10] clarified the nature of action of dental liners (bases) in the causation of NCS neurological and dermatological symptoms based on in vitro and in vivo studies, in part, and provided the history of 3 NCS patients who have recovered following treatment thus establishing a cause-effect relationship; pre- and post-recovery pictures were included. Various versions of this major publication were subsequently published elsewhere $[11,12,15]$. The author has established a causal relationship with dental toxins, as well as with other less frequent toxic exposures, developed a protocol, and successfully treated patients. All patients that have followed the author's protocol had invariably recovered. Most people have had dental work. Many have various degrees of sensitivity to some dental 
materials to which their bodies manifest varied intensities of allergic reactions. This epidemic-in-disguise has been routinely misdiagnosed by medical professionals who often label patients as delusional because of their unfortunate description of their neurological symptoms (actually caused by nerve damage) as having been caused by parasite infections. Amin [13] specifically addressed this issue while discussing the clinical history of 24 NCS patients that have been misdiagnosed by other medical professionals. Of these patients, 7 who have followed the author's protocol and completed treatment by the time of publication have experienced full recovery. Amin [14] provided an annotated list of about 360 dental materials that have been involved in the causation of NCS symptoms in patients that the author has seen. Toxic ingredients common to all listed chemicals were classed in 4 categories. These categories are found in many more dental chemicals that were not reported previously [14] but existed in the author's data base and published later [20]. An overview of NCS [16] made special reference to organ system symptomology in 50 patients of both sexes and all age groups, misdiagnoses, storage organs, liners, drug involvement, incubation period, and recovery, with a detailed discussion of 5 relevant cases. Amin [17] further analyzed 18 new NCS cases that have been in various stages of treatment and recovery and have demonstrated that those patients that have followed and completed the author's protocol have invariably recovered. Among patients experiencing recovery, 15 have shared their experience and perspectives [18]. NCS symptoms stated by 166 patients upon first examination at PCI were reported [19]. These patients expressed how they felt then and the reasons for which they came to see us in the first place. The final listing of dental materials involved in NCS cases [20] included 644 dental products.

\subsection{Additional Notes about Toxic Dental Materials}

The active toxic ingredients established as the causal factors for NCS observed in many of the author's patients were researched using actual patient histories, interviews, symptomology, OSHA's MSDS sheets of dental materials used, and dental biocompatibility test results. The components in catalysts and bases of Dycal, Life and Sealapex were identified $[11,12]$ with an analysis of the nature of action of the included toxic ingredients causing observed dermatological and neurological symptoms in NCS patients. Reference was also made to Fynal, IRM, Sultan U/P, Gluma One Bond and Scotch Bond [11]. These dental products among the 644 listed in Amin [20] are now recognized as toxic based on patient's symptoms, MSDS forms, and blood biocompatibility test results. Toxicity and individual reactivity will vary from person to person depending on degree of sensitivity to the compounds which is related to the concentration of the toxic ingredient(s) in the product, amount of product used, the age at which incompatible dental materials were first used and the number of teeth involved [16]. That degree of sensitivity is established by a blood biocompatibility test for dental materials that the author contract out to a laboratory in Colorado. The toxic ingredients common to all 644 dental products reported [20] belong in four major categories: Zinc Oxide, Ethyltoluene Sulfonamide (especially in patients with allergy to sulfa and toluene), Titanium Dioxide and other metal compounds (especially in implant patients and others with allergy to metals), and Methacrylate compounds, as well as Calcium Hydroxide often thought to be harmless. The author's studies of the many NCS patients examined indicate that these ingredients are causally associated with their symptoms. When the source (cause) is removed by appropriate dental rehabilitation and detoxification, and other cleansing processes are properly implemented, the patient recovers (effect). It should be noted that while the 2012 list is far more comprehensive than the one reported earlier [14], it may not include all dental products in the market 
which continually change and increase. The author has concluded, however, that the listing of more dental compounds will not make the point any stronger at this time.

\subsection{Supporting Reports}

On some occasions, one comes across reports supporting the author's contention. For instance, Kal et al. [26] described an immediate hypersensitivity reaction associated with the mercury component of amalgam restorations. The author reported that the mercury from amalgam fillings induced an acute reaction which resulted in erythematous lesions, severe burning and itchy sensation and difficulty in breathing. The amalgam restorations were replaced with compatible composite restorations and the symptoms resolved. Follow-up skin patch test results indicated a very strong positive reaction to mercury. Rare instances of delayed, localized allergic reactions have been reported in the literature.

\subsection{What can Dentists do?}

Dentists should always attempt to identify the toxic elements in the dental products or implants that they use whether they are included in this list or not. They should examine the relevant OSHA-required MSDS forms especially when they embark on major dental procedures, and should insure their biocompatibility to patients' immune systems. Please note that toxicity and individual reactivity will vary from person to person and that there is no one product that is "safe" for everyone. What is safe for one person may be toxic for another.

\subsection{A Case History}

Many case histories have been published in [10-13, $15-18,21,22,25]$. The following case of GC, a 50 year old white female from California is new and perhaps the most comprehensive. It is included to make a case for NCS and to give hope for Morgellons sufferers who have lost hope in a resolution through the conventional medical community. GC first came to see us at PCI in May, 2010 with full blown classical neurological and dermatological NCS symptoms (Fig. 1-5). She was clearly NOT a case of delusionary parasitosis. Her more recent dental work included restoration of tooth No. 14 (a 30-year old root canal) on December 11, 2006. Surgical and non-surgical endodontic treatments of tooth No. 14 were completed on the same date. This consisted of retreatment and apicoectomy with MTA Retrofill procedures. On December 22, 2006 she had a crown porcelain-fuse high noble and a build up with pins on the same tooth. On January 25, 2007 she had an amalgam filling in tooth No. 2. These dental events immediately preceded the onset of her recent symptoms so graphically described in her anguished personal narrative of her history and experience presented on her web site http://morgellonscentral.wordpress.com.

"For years I had searched for answers to mystifying symptoms I suffered: painful skin rashes, fatigue, body aches, itchy crawling sensation on my skin and in my body that began at 14 years old. Most doctors assigned my symptoms to 'psychosomatic' since most blood tests were 'text book' normal. The skin rashes were diagnosed as acne that would come and go. During my mid 30's the painful skin rashes continued and breakouts would appear anywhere including my forearms, scalp and ankles, not usually places for acne. In addition, I was in incredible physical pain and so fatigued. In 1999, at 36, I was diagnosed with fibromyalgia. In the late 90 's there was no known cure so I researched the internet for information. I went on a detox regimen including vitamins, supplements and sunshine; vitamin D which is now used to relieve fibromyalgia symptoms. After about 2 years I recovered and was doing well, but as I reached 40 , the skin rashes reappeared.

The "adult acne" would not heal. I noticed white stretchy "splinters" that could be pulled out of the sores. Tests proved negative for microorganisms including bacteria. I was prescribed antibiotics and antifungals 
but without a marked improvement in my condition. I was severely exhausted all the time again. I was a mother, a wife, a business owner, and worked full-time, but secretly I felt awful and ashamed. An internet search in 2008 showed that Morgellons was so much like what I experienced. In May of 2010, I became aware of mainstream medicine and CDC's (Center for Disease Control and Prevention) statements that Morgellons was only "delusional parasitosis". However, my internet search for doctors treating Morgellons yielded one that sounded credible, Dr. Omar Amin at Parasitology Center, Inc. (PCI), Scottsdale, Arizona. He wrote intelligently on the subject and had patient interviews of illness and recovery. I made an appointment.

On appointment day, I explained my symptoms to him and my skin condition was visible to the naked eye (Fig. 1-5). He concluded it was Morgellons which he terms NCS (Neuro-cutaneous Syndrome). He explained that my body's $\mathrm{pH}$ and 'fighting' ability have been altered by intimate exposure to toxins, most likely from my dental work but, a blood biocompatibility test was needed for confirmation. The rash was a reaction to the toxins trying to exit my skin and offered an inviting home for secondary infections and mine was a plant mold.

A dental biocompatibility blood test, one of the PCI protocol items that Dr. Amin recommended, revealed that at the very least, my body is indeed incompatible with metals, especially heavy metals, many of which are commonly used in medicine, dental work, cosmetics, toiletries, food and drinks. My braces, at 14 years old, were nickel and all my fillings were Mercury amalgam (amalgam also contains copper) as well as two root canals. Some were $30+$ years old. A PCI detox protocol of homeopathic products and vitamins was recommended. Dr. Amin assured me that as the toxins diminish so will the itching and crawling feelings, and the sores will heal. I was ecstatic. I had answers.

I had the fillings removed by a dentist who specializes in the removal of mercury amalgam. When amalgam fillings were ground down they 'smoked.' The fumes contain mercury and are breathed in. This is a very toxic exposure to mercury for anyone and can have terrible effects on those not sufficiently protected.

I had a tremor in my left hand for about 5 years. I could not draw a number 6 without scribbles. I didn't tell a soul, I was only 40 , but it stopped the very day I had my last filling removed. Why, because mercury is a fabulous conductor of electricity and interferes with the body's natural electrical currents. I was instructed to have mercury amalgam fillings removed in stages, as horrid reactions are possible doing too many at once. I didn't listen. I had all fillings removed in one day. Luckily, I experienced no backlash so, without hesitation, the following week I had both root canals removed in one visit.

The oral surgeon didn't outfit me with a mask or use a special vacuum/pump to protect me or himself from toxic fumes. It didn't occur to me that it was necessary since the teeth were being pulled out. Unfortunately, they didn't just pop right out. He had to grind and saw at the teeth to break them up so they would come out. The oral surgeon looked so pale after digging and grinding to get the root canals out of my head. He sent me off with antibiotics and oxycodone. Within hours I developed a fever. The itching and crawling on my face and body was beyond anything I had experienced. The office called to see how I was doing. Upon hearing my symptoms, they said to stop taking the oxycodone, "you're allergic". So, I did.

I spent the next two weeks in bed, too weak to eat, feverish and covered in sores. My neck on both sides, just under my ears had open sores the size of a quarter that was draining and my face peeled for 2 months with a new symptom, muscle jerks. My legs would "kick" involuntarily, like a heroin addict. I was in a horrid state. What I found out later is that I was poisoned by the fumes from my tooth removal. Root canals can have mercury, copper, silver, aluminum and nickel (base noble). As he ground and drilled, fumes were released. He was probably not doing so well either but, 
I will never know.

Amazingly, I got through those days and slowly resumed the PCI recovery protocol (detox body from heavy metals and dental chemicals, among other steps). I didn't go in public for about 2 months. I was weak and my face was raw, peeling and oozing. The detox is uncomfortable in the beginning. Itching and crawling sensations got worse. My skin lesions (Fig. 1-5) were so painful. The pin pricking sensations and muscle jerks in my legs were awful. I understood later that symptoms become more extreme at the beginning of detoxification. That was normal. I took sedatives to get sleep; Benadryl and I became good friends. Perspiration would smell like metal, like moldy towels and my face sweat, a hallmark of mercury poisoning. I have had to pause the detox at times for financial reasons, sometimes just because it was uncomfortable and I had so much going on that I couldn't endure feeling sick too. In addition, I have shed $70 \mathrm{lbs}$. of fat. Every level of weight reduction triggered skin rashes, i.e., stomach, back, arms, fatigue, flu like symptoms and skin sensations. Toxins store in fat cells, so losing fat will release toxins. Because I have a known allergy to almost all heavy metals, a buildup of copper or aluminum, which is necessary for our healthy metabolism can be toxic for me. I stay on a maintenance recovery protocol and eat clean and simple to feel my best. This helps me live in the real world without fear of environmental assaults.

I am now back to my normal healthy state (Fig. 6). Gone are the days that I would fill up the bath tub with Epsom salts and hot water (be sure to drink water while soaking as Epsom baths are dehydrating) and submerge everything but my nose holes to soothe the itching and crawling. The itching and crawling are gone. The muscle twitches and tremors are gone. Gone are the days being covered from head to toe with an oozing rash and gone is the plant mold. Gone is excruciating fatigue. I now walk 10-15 miles, workout 3 times a week, and for fun, I kayak, canoe, paddle board, or hike and work, no exhaustion! I am feeling wonderful. Now
I desire to share what I have learned on this journey and dispel myths surrounding Morgellons/NCS."

\subsection{Forward}

The author hopes that this presentation, and follow up by practitioners, will help the proponents of the delusionary parasitosis convention to revise their ideas and take a fresh look at alternative approaches that work, instead of dismissing their patients' plight thus adding insult to injury. Publications by Amin in the following references list can be readily downloaded from the author's web site www.parasitetesting.com.

\subsection{Recommendations}

The author recommends that dentists research the OSHA required MSDS sheets for proposed dental materials to be used, and match blood bio-compatibility test results for dental materials (contracted to a Colorado laboratory) with patients' dental history before embarking on, at least major, dental procedures. It is a moral responsibility that dentists as well as surgeons use that approach, especially in cases of implants. Labeling their patients as crazy with prescriptions of anti-anxiety drugs instead of doing the hard work is unconscionable. The author's protocol works.

\section{Conclusions}

The author has concluded that the symptoms of crawling and pin-pricking confused with "delusionary parasitosis" are caused by toxicity from exposure to chemicals incompatible with host immune system, e.g., dental materials that alter the propagation of normal nerve impulses. The author also concludes that external parasites/organisms including springtails (Collembola), other arthropods, bacteria, and fungi represent only opportunistic infections of skin sites compromised by the toxicity disorder involving the elimination of toxins through the skin. The author has described a new pathological disorder, Neuro-cutaneous Syndrome (NCS) to which justified 
"delusional parasitosis" and Morgellons disease cases could be assigned. The author has developed a protocol for the resolution of the symptoms of NCS cases. When followed to the letter, all symptoms of NCS, conventionally and conveniently called delusional parasitosis, are invariably and irreversibly resolved.

\section{Competing interests}

The author declares that he has no competing interests.

\section{Acknowledgments}

I am grateful to Miss Kyrie Carpenter and Dr. Richard Heckmann, Biology Department, Brigham Young University, Provo, Utah for the management and editing the plate of figures.

\section{References}

[1] Aw, D., Thong, J., and Chan, H. 2004. "Delusional Parasitosis: Case Series of 8 Patients and Review of the Literature." Ann Acad Med 33: 89-94.

[2] Bak, R., Tumu, P., Hui, C., Kay, D., Burnett, J., and Peng, D. 2007. "A Review of Delusions of Parasitosis, Part 1: Presentation and Diagnosis." Cutis 82: 123-130.

[3] Donabedian, H. 2007. "Delusions of Parasitosis." Clin Infect Dis.45: 131-134.

[4] Driscoll, M. S., Rothe, M. J., Grant-Kels JM, M. S., Hale, M. S. 1993. "Delusional Parasitosis: A Dermatologic, Psychiatric, and Pharmacologic Approach.” J Amer Acad Dermatol. 29: 1023-1033.

[5] Hinkle, N. C. 2000. "Delusionary Parasitosis." Amer Entomol. 46: 17-25.

[6] Lyell, A. 1983. "Delusions of Parasitosis." Brit $J$ Dermatol. 108: 485-499.

[7] Trabert, W. 1995. "100 Years of Delusional Parasitosis. Meta-analysis of 1,223 Case Reports." Psychopathol 28: 238-246.

[8] Shelomi, M. 2013. "Evidence of Photo Manipulation in A Delusional Parasitosis Paper." J Parasitol. 99: 583-585.

[9] Lyons, E. T. 2013. "Delusional Parasitosis." J Parasitol. Newsletter 35: 7-11.

[10] Amin, O. M. 2003. "On the Diagnosis and Management of Neuro-cutaneous Syndrome (NCS), A Toxicity Disorder from Dental Sealants." Explore 12: 21-25.
[11] Amin, O. M. 2004. "Dental Sealant Toxicity: Neurocutaneous Syndrome (NCS), A Dermatologicaland Neurological Disorder." Holistic Dent Assoc. J 2004 (1): $1-15$.

[12] Amin, O., M. 2004. "On the Diagnosis and Management of Neuro-Cutaneous Syndrome, A Toxicity Disorder from Dental Sealants." Calif Dent Assoc. J 32: 657-663.

[13] Amin, O., M. 2004. "On the Course of Neurocutaneous Syndrome (NCS) and Its Pseudodiagnosis by Medical Professionals." Explore 13: 4-9.

[14] Amin, O., M. 2005. "Dental Products Causing Neuro-cutaneous Syndrome (NCS) Symptoms in NCS Patients." Explore 14: 57-64.

[15] Amin, O., M. 2006. "On the Diagnosis and Management of Neuro-cutaneous Syndrome (NCS) A Toxicity Disorder from Dental Sealants." Townsend Letter 276: 85-90.

[16] Amin, O., M. 2006. “An Overview of Neuro-cutaneous Syndrome (NCS) with A Special Reference to Symptomology." Explore 15: 41-49.

[17] Amin, O., M. 2007. "The Face of Neuro-cutaneous Syndrome (NCS): New Cases, Recovery, and Perspectives." Explore 16: 54-64.

[18] Amin, O., M. 2009. "Recovery from Morgellons and Neuro-Cutaneous Syndrome (NCS): Patients Perspectives." Explore 18: 25-33.

[19] Amin, O., M. 2010. "In Their Own Words OR: Symptoms of Morgellons and Neuro-cutaneous Syndrome (NCS) upon First Examination at the Parasitology Center, Inc. (PCI).” Explore 19: 24-31.

[20] Amin, O., M. 2012. "A Comprehensive Study of Dental Materials and Their Toxic Ingredients Associated with Neuro-cutaneous Syndrome (NCS) and Morgellons, with Notes on Research Background." Explore 21: 14-23.

[21] Amin, O., M. 2013. "The Misdiagnosis of 'Delusional Parasitosis'." J. Parasitol, Photon 104: 139-144.

[22] Amin, O., M. 2001. "Neuro-cutaneous Syndrome (NCS): A New Disorder." Explore 10: 55-56.

[23] Hunter, G. W., Frye, W. W., and Schwartzwelder, J. C. 1960. A Manual of Tropical Medicine. Philadelphia: W. B. Saunders.

[24] Scott, H. G., Wiseman, J. S., and Stojanovich, C. J. 1962. "Collembola Infesting Man." Ann Entomol Soc Amer. 55: 528-530.

[25] Amin, O., M. 1996. "Facial Cutaneous Dermatitis Associated with Arthropod Presence." Explore 7: 62-64.

[26] Kal, B. I., Evcin, O., Dundar, N., Tezel, H., and Unal, I. 2008. "An Unusual Case of Immediate Hypersensitivity Reaction Associated with An Amalgam Restoration." Brit Dent J 205: 547-550. 